\title{
Region-Dot Conversion Fusion Algorithm and Application
}

\author{
Zhouxing Fu, ${ }^{1}$ Mei Wang, ${ }^{1}$ Jingyi Du, ${ }^{1}$ Hsiung-Cheng Lin, ${ }^{2}$ \\ Ningbo $\mathrm{Xu},{ }^{1}$ and Hongyang $\mathrm{Zan}^{1}$ \\ ${ }^{1}$ College of Electric and Control Engineering, Xian University of Science and Technology, Xian 710054, China \\ ${ }^{2}$ Department of Electronic Engineering, National Chin-Yi University of Technology, Taichung 41170, Taiwan \\ Correspondence should be addressed to Mei Wang; wangm@xust.edu.cn
}

Received 26 February 2014; Revised 26 April 2014; Accepted 26 April 2014; Published 28 May 2014

Academic Editor: Her-Terng Yau

Copyright (C) 2014 Zhouxing Fu et al. This is an open access article distributed under the Creative Commons Attribution License, which permits unrestricted use, distribution, and reproduction in any medium, provided the original work is properly cited.

\begin{abstract}
The interior decoration materials and the new furniture using formaldehyde, ammonia, and other poisonous substances are known as the main sources of indoor air pollutions. However, it is still a big challenge to estimate accurately the overall air quality by using the current measuring tools. Accordingly, the region-dot fusion (RDF) algorithm is proposed to evaluate the air quality in this paper. For the conversion from a region to a dot, the region-dot function is firstly defined as the summation of the belief function and the weighted width of the belief interval. In the RDF algorithm, the belief intervals of the two sensors with the basic probability functions are calculated based on the measurements of formaldehyde sensor and ammonia sensor. Then, the belief intervals are converted to the specific values. After the computation of collision degree and combination, the pollution level represented by a belief interval with the maximum probability is selected as the outcome of fusion decision. Compared with the weighted fusion algorithm and D-S evidence reasoning method, it is experimentally proved that the RDF algorithm can improve the separability of the belief intervals of the belief functions. Also, the evidence collision degree is decreased dramatically.
\end{abstract}

\section{Introduction}

Environment pollution is one of the most severe problems on the earth. Among them, indoor air pollution produced from decoration and new furniture is also of concern but easily ignored. Usually, the decoration materials and the new furniture contain formaldehyde, ammonia, and other poisonous components. It is quite clear that the indoor air pollution exists within our living surrounding widely. Aiming at these poisonous substances, the development of the effective air quality estimation method is a crucial research topic.

Based on the recent publications, scientists [1] used the differential optical absorption spectroscopy, Fourier transform infrared spectroscopy, and ceilometer to interpret and estimate the air quality of the surrounding. However, these studies were focused exclusively on the outdoor air quality estimations so that they may not be suitable for indoor case.

For the indoor air quality study, researchers [2] developed the preconcentrator for the detection of trace amounts of formaldehyde. In addition, Akshath et al. [3] detected formaldehyde in food samples by the enhanced chemiluminescence. Although these methods may detect the formaldehyde efficiently, the gas mixture which also pollutes the indoor air was not considered. Panagopoulos et al. [4] studied the computational fluid dynamics simulation of volatile organic compounds and indoor air pollution dispersion. Unfortunately, the evidence collision degree and imprecision problems were not revealed in these works.

To solve the collision and imprecision problem, the neural network with Dempster-Shafer evidential reasoning [5] was applied for data fusion. However, the neural network required a large number of training samples which were usually difficult to be obtained. Based on the expert knowledge, Bayes networks [6] were used to calculate the posterior probability. Scientists [7] defined the uncertainty of a hierarchical quotient space structure and then proposed the information entropy sequence to complete the reasoning. These methods have the advantage for the prediction in collision case, but it may suffer from the differentiation between the collision and the unknown one.

In advanced study, Wang et al. [8] used the improved Dempster-Shafer (D-S) evidential reasoning to evaluate the 
quality level of the indoor air efficiently. The uncertainty problem has been studied, but the evidence collision problem was not worked out. In another case, the adaptive-weighting fusion (AWF) method [9] could employ efficiently the original data without the experience knowledge, but it lacks adequate capability in the collision problem.

Opposite to the algorithm from pixels to region [10] and making use of advantage of the multiple sensor fusion $[11,12]$, we developed the region-dot fusion (RDF) algorithm for the air quality estimation in this paper. In Section 1, the literature reviews about related study are provided. In Section 2, the background about the RDF algorithm and the indoor air quality estimation are described. It includes the basic probability function, belief function, D-S combination rule, and adaptive weighted fusion (AWF) algorithm. In Section 3, we illustrate how the system architecture is erected. It includes the hardware scheme, software profile, and RDF algorithm description. In Section 4, the experiments and comparison analyses are given to demonstrate the proposed RDF algorithm for the indoor air quality estimation. The conclusion is given in Section 5.

\section{Background}

In this section, we introduce the theoretical background of RDF. It includes the basic probability function, the belief function, the evidence combination rule, and the AWF method.

2.1. Basic Probability Function. In the evidential reasoning theory $[13,14]$, proposition $A$ is an element of identification frame $\Gamma$, and the elements satisfy the incompatible condition. The basic probability function $P(A)$ of the proposition $A$ can be obtained by analyzing the evidence. It denotes the accurate belief degree of proposition $A$ and represents the direct support to the proposition $A$. The function $P(A)$ is a mapping from a set $2^{\mathrm{\Gamma}}$ to $[0,1]$. If the function $P(A): 2^{\Gamma} \rightarrow$ $[0,1]$ satisfies the following conditions:

$$
\begin{gathered}
P(\Phi)=0 \\
P(A) \geq 0 \\
\sum_{A \in 2^{\Theta}} P(A)=1 \\
\forall A \in 2^{\Gamma}
\end{gathered}
$$

then $P(A)$ is the basic probability function of proposition $A$ and $\Phi$ is an empty set and $\Theta$ belongs to the integer.

2.2. Belief Function. For a given basic probability assignment function $P$ and an arbitrary proposition $A \in 2^{\Gamma}$, the corresponding belief function $\operatorname{Bel}(A)$ of proposition $A$ is

$$
\operatorname{Bel}(A)=\sum_{X_{i} \subseteq A} P\left(X_{i}\right), \quad i=1,2, \ldots, N,
$$

where $X_{i}$ is the $i$ th component which is included in $A$.

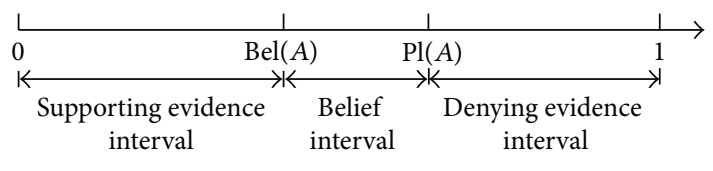

FIGURE 1: Evidence intervals.

Function $\operatorname{Bel}(A)$ represents the total belief degree of proposition $A$

$$
\begin{aligned}
& \operatorname{Bel}(\Phi)=0 \\
& \operatorname{Bel}(\Omega)=1,
\end{aligned}
$$

where $\Omega$ is the set of the total propositions, and $\Phi$ is an empty set.

The plausibility function $\operatorname{Pl}(A)$ is

$$
\operatorname{Pl}(A)=1-\operatorname{Bel}(\bar{A})
$$

where $\bar{A}=\Gamma-A$.

Function $\mathrm{Pl}(A)$ is the nonnegation function. It denotes that the belief degree of the proposition $A$ is not denied. Function $\mathrm{Pl}(A)$ is the sum of the basic probability assignment functions of the sets which have the intersection with the proposition $A$.

If the evidence denies the proposition $A$, there is

$$
\operatorname{Pl}(A)=0 \text {. }
$$

If the evidence does not deny the proposition $A$, there is

$$
\begin{gathered}
\operatorname{Pl}(A)=1 \\
\operatorname{Pl}(A) \geq \operatorname{Bel}(A) .
\end{gathered}
$$

The region $[\operatorname{Bel}(A), \mathrm{Pl}(A)]$ represents the belief interval $[15,16]$ of the proposition $A$. If a subset of the identification frame $\Gamma$ is the proposition $A$, and $p(A)>0$, then the subset $A$ of frame $\Gamma$ is the focal element of the belief function $\operatorname{Bel}(A)$. Evidence intervals are shown in Figure 1.

2.3. D-S Combination Rule. According to D-S combination rule [17], if $P_{1}$ and $P_{2}$ are the belief functions in the same identification frame $\Gamma$, and the focal elements are $U_{1}, U_{2}, \ldots, U_{k}$ and $V_{1}, V_{2}, \ldots, V_{k}$, respectively, then the jointed belief function $P(W): 2^{\Gamma} \rightarrow[0,1]$ is written bellow:

$$
P(W)= \begin{cases}\frac{1}{1-C} \sum_{U_{i} \cap V_{j}}\left[P_{1}\left(U_{i}\right) P_{2}\left(V_{j}\right)\right], & U_{i} \cap V_{j} \neq \Phi \\ 0, & U_{i} \cap V_{j}=\Phi,\end{cases}
$$

where

$$
\begin{gathered}
W=U_{i} \cap V_{j} \\
C=\sum_{U_{i} \cap V_{j}=\Phi} P_{1}\left(U_{i}\right) P_{2}\left(V_{j}\right) .
\end{gathered}
$$


The denominator of (7) is a modifying factor, which is used to normalize the coefficient. In order to avoid the fact that the nonzero probability is assigned to the empty set and then the deserted belief degree of the empty set is assigned to the nonempty set, the factor $(1-C)$ was employed. The parameter $C$ reflects the collision degree between the evidences during the fusion process, $0 \leq C \leq 1$. The larger the parameter $C$ is, the severer the collision degree would be. If the parameter $C$ is close to 1 , the unreasonable result would appear likely, and the fusion decision would be opposite to the intuition. If $C$ is equal to 1 , the D-S theory could not be used for fusion.

D-S evidence combination rule provides the method to combine two evidences. For more evidences combination, we may use (7) repeatedly to make the pairwise combinations until the solution is satisfactory.

2.4. Adaptive Weighted Fusion Algorithm. In AWF method, the optimal function is the least mean-squared error function of the measurement of a sensor. The fusion value of the measurement of a sensor is obtained by finding the corresponding weights. In this process, the AWF method is used [18-22].

Assume that the $n$ measurements of a sensor, $X=$ $\left[X_{1}, X_{2}, \ldots, X_{n}\right]$, are independent of each other. The variances of the sensors are $\sigma_{1}^{2}, \sigma_{2}^{2}, \ldots, \sigma_{n}^{2}$, respectively. The weighted factors are introduced as $\omega=\left[\omega_{1}, \omega_{2}, \ldots, \omega_{n}\right]$

$$
\sum_{i=1}^{n} \omega_{i}=1
$$

The detailed steps of the AWF algorithm are described as follows [18, 23-25].

Firstly, calculate the mean value $\bar{X}_{i}(k)$ of sensor $i$ using the $n$ measurements

$$
\bar{X}_{i}(n)=\frac{1}{n} \sum_{j=1}^{n} X_{j} \quad(i=1,2, \ldots, k),
$$

where $X_{j}$ is the $j$ th measurement; $n$ is the total number of measurements of a sensor; and $i$ represents the $i$ th sensor among the $k$ sensors.

Secondly, find each variance $\sigma_{i}^{2}[26,27]$ of each sensor $i$

$$
\sigma_{i}^{2}=E\left[(X-\bar{X})^{2}\right] .
$$

Thirdly, calculate the optimal weighted factor $\omega_{i}$

$$
\omega_{i}=\frac{1}{\sigma_{i}^{2} \sum_{i=1}^{2}\left(1 / \sigma_{i}^{2}\right)} .
$$

Finally, calculate the fusion value $\widehat{\bar{X}}[28]$

$$
\widehat{\bar{X}}=\sum_{i=1}^{k} \omega_{i} \bar{X}_{i}(n) .
$$

Consequently, the AWF value $\widehat{\bar{X}}$ is obtained by (13).

\section{System and RDF Algorithm}

The proposed system contains the hardware scheme and the software profile. The RDF algorithm is used in the system.

3.1. Hardware Scheme. In Figure 2, the system hardware includes two signal conditioning circuits for the formaldehyde sensor and ammonia sensor, TMS320LF2407A controller, communication circuit between the monitor and the controller, LED display circuit, and alarming circuit using acoustic and optical circuit. Figure 3 shows a photo of the real system.

The outputs of the formaldehyde sensor and ammonia sensor are the voltage signals. The signal conditioning circuits magnify the voltages to $0-3.3 \mathrm{~V}$ to match the controller TMS320LF2407A interface. The controller has an A/D converter with 10 bits. The analog data is converted to the digital data by the $\mathrm{A} / \mathrm{D}$ converter.

The RDF algorithm is used in the controller to obtain the air quality estimation result. Consequently, the fused air quality value can be contrasted with the standard level.

If the fused concentration of the poison gases is beyond the standard level, the alarming circuit will be activated immediately. Meanwhile, the concentration values and the fused result are sent to the monitor by RS-232 for displaying.

3.2. Software Profile. The system software consists of the VB language program, the assemble language program, and $\mathrm{C}$ language program.

VB language program is designed for the monitoring purpose, including real-time data transmission, data storage, and gas concentration display. The assemble language program and $\mathrm{C}$ language program are written in the controller. It implements the functions such as the system initiation, interruption sampling, acoustic and optical alarming, and data transmission. Figure 4 shows the flow chart of air quality estimation.

3.3. RDF Algorithm Description. Belief interval represents the believable degree of a proposition that is located in a region. It also means that the proposition has a certain degree of uncertainty although it has a certain degree of certainty. The uncertainty would make the problem complicated so that it would be difficult to be solved. To eliminate the uncertainty of a proposition and make the problem simple, belief interval should be converted to specific value. In the proposed RDF algorithm, the conversion is firstly defined from a region to dot value. Then, the RDF algorithm is developed by the combination of the conversion formula with the D-S theory. The RDF algorithm scheme is shown in Figure 5.

It can be seen that the measurements of the two sensors are converted after the corresponding belief degree values are assigned, and then the D-S combination rule is processed. Finally, the decision is thus made. 


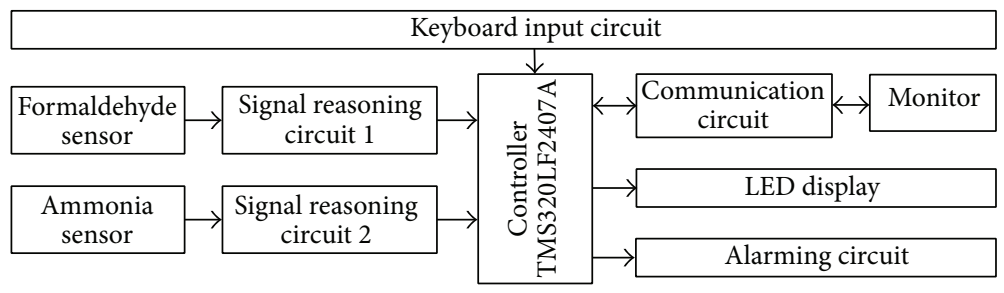

FIGURE 2: Hardware scheme of the air quality estimation system.

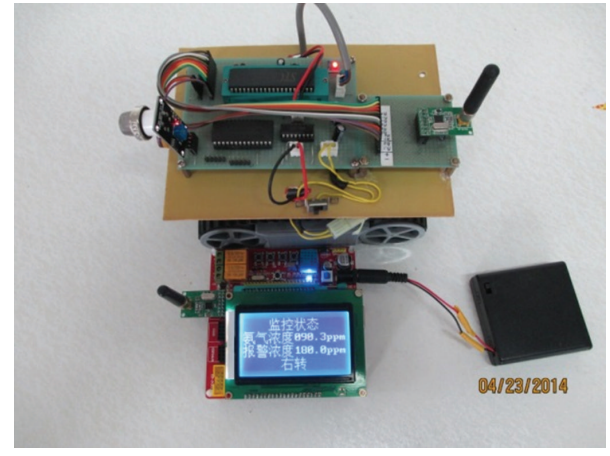

Figure 3: A photo of the real system.

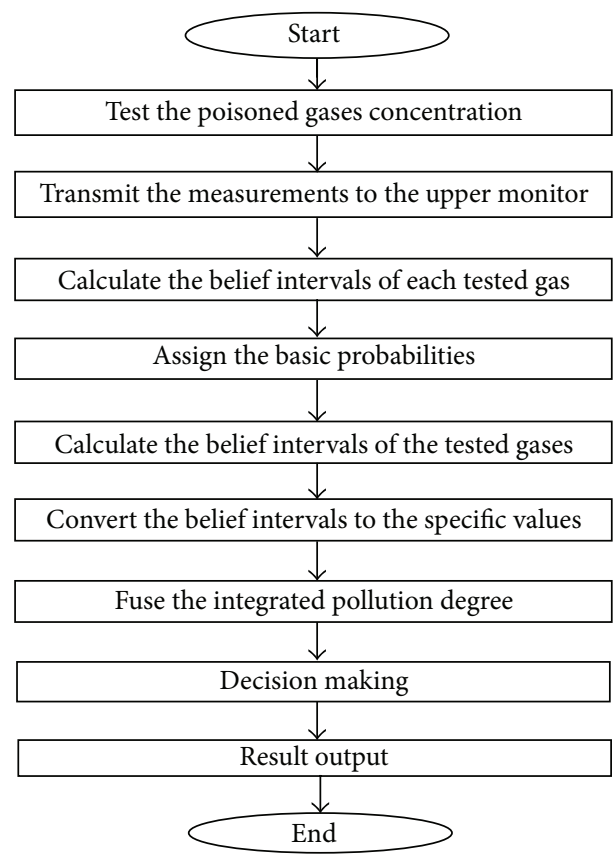

FIGURE 4: Flow chart of air quality estimation.

Definition of RD Function. The conversion formula is defined from a region to a dot as the summation of the belief function and the weighted width of the belief interval

$$
m(A) \triangleq \operatorname{Bel}(A)+\alpha[\operatorname{Pl}(A)-\operatorname{Bel}(A)],
$$

where $A$ is a proposition, $\operatorname{Bel}(A)$ and $\operatorname{Pl}(A)$ denote, respectively, the lower and upper probabilities of the belief interval of a proposition, and $\alpha$ is a coefficient, $0 \leq \alpha \leq 1$.
$R D F$ algorithm is described as follows.

Step 1. Input the measurements $U_{i}$ and $V_{i}$ of the formaldehyde and ammonia sensors.

Step 2. Assign the basic probability function values and the belief interval.

Step 3. Calculate the belief function and plausibility function of the belief intervals of the measurements to construct the belief intervals

$$
\begin{aligned}
& \operatorname{Bel}(U)=\sum_{U_{i} \subseteq U} P\left(U_{i}\right) \\
& \operatorname{Pl}(U)=1-\operatorname{Bel}(\bar{U}) \\
& \operatorname{Bel}(V)=\sum_{U_{i} \subseteq U} P\left(V_{i}\right) \\
& \operatorname{Pl}(V)=1-\operatorname{Bel}(\bar{V}),
\end{aligned}
$$

where $U$ and $V$ are, respectively, the measurement set of sensor formaldehyde and sensor ammonia; $U_{i}$ and $V_{i}$ are, respectively, the $i$ th measurement of sensor formaldehyde and sensor ammonia.

Step 4. Convert the belief interval to a specified value by using the RD function

$$
\begin{aligned}
& m(U)=\operatorname{Bel}(U)+\alpha[\operatorname{Pl}(U)-\operatorname{Bel}(U)] \\
& m(V)=\operatorname{Bel}(U)+\alpha[\operatorname{Pl}(V)-\operatorname{Bel}(V)] .
\end{aligned}
$$

Step 5. Calculate the evidence collision degree parameter $C$

$$
C=\sum_{U_{i} \cap V_{j}=\Phi} P_{1}\left(U_{i}\right) P_{2}\left(V_{j}\right) .
$$

Step 6. Calculate the combination result $P$

$$
P(U, V)= \begin{cases}\frac{1}{1-C} \sum_{U_{i} \cap V_{j}=W}\left[P_{1}\left(U_{i}\right) P_{2}\left(V_{j}\right)\right], & W \neq \Phi \\ 0, & W=\Phi,\end{cases}
$$

where $P$ means the probability that the fusion result belongs to a belief interval $B_{i}$.

Step 7. Make the fusion decision

$$
\begin{gathered}
P \in B_{i} \\
P_{i}=\max \left\{I_{j}, j=1,2, \ldots, M\right\},
\end{gathered}
$$

where $M$ is the total number of the belief intervals $B_{i}$. 


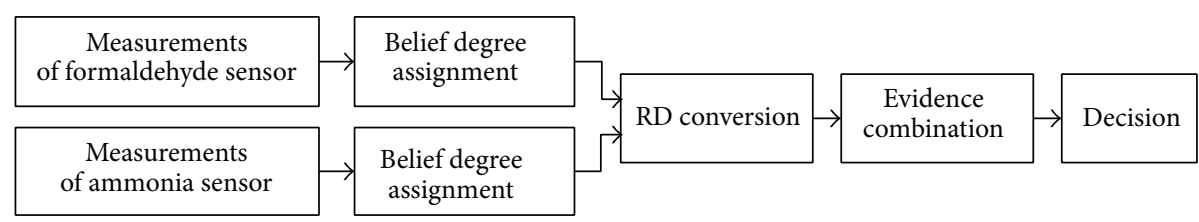

FIGURE 5: Scheme of region-dot fusion algorithm.

The proposed RDF algorithm calculates the belief intervals of the two sensors with the basic probability functions based on the sensor's measurements of formaldehyde and ammonia sensors. Then, the belief intervals are converted to the determined belief values. After the combination, the belief interval with the maximum probability is selected as the outcome of fusion decision.

\section{Experimental Results and Discussions}

To verify the effectiveness of the proposed RDF algorithm for indoor air quality estimation, the experiments were carried out in this section.

4.1. Data Collection. Generally, the standard for indoor air pollution level can be defined in Table 1, where the pollution index $I$ is used for classification of pollution level. The 4 intervals are defined as

$$
\begin{array}{ll}
B_{1}=[0,0.1), & B_{2}=[0.1,0.3), \\
B_{3}=[0.3,0.5), & B_{4}=[0.5,1] .
\end{array}
$$

They represent the pollution levels in "no pollution," "light pollution," "medium pollution," and "serious pollution," respectively.

In this experiment, the data is collected 10 times using formaldehyde and ammonia sensors. The measurement results with basic probabilities are listed in Tables 2 and 3 . Please note that the unit "ppm" is the gas concentration, indicating the contamination volume number in the million volumes.

4.2. Results with AWF Algorithm. The data shown in Tables 2 and 3 was also used to perform the adaptive weighted fusion. The mean values of formaldehyde and ammonia are calculated below:

$$
\begin{aligned}
& \text { Formaldehyde : } \bar{X}_{1}(n)=\frac{1}{10} \sum_{j=1}^{10} X_{j}=0.5422 \\
& \text { Ammonia : } \bar{X}_{2}(n)=\frac{1}{10} \sum_{j=1}^{10} X_{j}=0.6125
\end{aligned}
$$

The variances and the weighted factors of formaldehyde and ammonia are shown in Table 4.
TABLE 1: Indoor air pollution level.

\begin{tabular}{lc}
\hline Pollution level & Pollution index $I$ \\
\hline No pollution & $I<0.1$ \\
Light pollution & $0.1 \leq I<0.3$ \\
Medium pollution & $0.3 \leq I<0.5$ \\
Serious pollution & $I \geq 0.5$ \\
\hline
\end{tabular}

Then, the AWF value is

$$
\begin{aligned}
\widehat{\bar{X}} & =\sum_{i=1}^{k} \omega_{i} \bar{X}_{i}(n)=0.6582 \times 0.5422+0.3418 \times 0.6125 \\
& =0.5662>0.5 .
\end{aligned}
$$

According to the standard in Table 1, the AWF value indicates that the indoor air pollution belongs to the serious pollution level.

4.3. Results with RDF Algorithm. Based on (15) and (16), the upper boundary and lower boundary of the probability of each evidence interval can be obtained by using the data in Table 2. Further, the probability intervals of formaldehyde concentrations are found to be $[0,0.05],[0,0.05]$, $[0.28,0.33]$, and $[0.67,0.72]$, respectively.

In addition, the ammonia measurements were processed based on (17) and (18) by using the data in Table 3. The probability intervals of ammonia concentrations are obtained to be $[0,0.05],[0,0.05],[0.19,0.24]$, and $[0.76,0.81]$, respectively.

The basic belief intervals of formaldehyde and ammonia are shown in Table 5. Accordingly, the dot value based on (7) by using the data in Table 5 can be obtained in Table 6, where " $C$ " represents the collision degree. Using the belief values of formaldehyde and ammonia shown in Table 6 , the combinations of formaldehyde and ammonia are achieved in Table 7. Finally, the fusion process can be done completely, and its result is shown in Table 8 .

Before the RD conversion fusion, as can be seen in Table 8, the basic belief function value of formaldehyde for level of "serious pollution" is 0.68 , larger than the values of the other classes of $0.1,0.1$, and 0.29 . On the other hand, the basic belief function value of ammonia for level of "serious pollution" is 0.77 , larger than the values of the other levels of $0.01,0.01$, and 0.20 .

After the RD conversion fusion, the basic belief function value becomes 0.8998 , larger than the values of the other classes of 0.0002, 0.0002, and 0.0996. Consequently, it is 
TABLE 2: Indoor formaldehyde measurement belief intervals and probabilities.

\begin{tabular}{lccc}
\hline $\begin{array}{l}\text { Measurement } U_{i} \\
(\mathrm{ppm})\end{array}$ & $\begin{array}{c}\text { Belief interval }=0.95, n=10 \\
\text { basic probability } m\left(U_{i}\right)=0.95 / n\end{array}$ & $\begin{array}{c}\text { Possible overall interval of truth value } U_{\text {it }} \\
(\mathrm{ppm})\end{array}$ & $\begin{array}{c}\text { Basic probability } m\left(U_{\text {it }}\right) \\
\text { of truth value } U_{\text {it }} n=10 \\
m\left(U_{\text {it }}\right)=0.5 / n\end{array}$ \\
\hline 0.501 & 0.095 & {$[0,1]$} & 0.005 \\
0.498 & 0.095 & {$[0,1]$} & 0.005 \\
0.589 & 0.095 & {$[0,1]$} & 0.005 \\
0.579 & 0.095 & {$[0,1]$} & 0.005 \\
0.657 & 0.095 & {$[0,1]$} & 0.005 \\
0.603 & 0.095 & {$[0,1]$} & 0.005 \\
0.527 & 0.095 & {$[0,1]$} & 0.005 \\
0.472 & 0.095 & {$[0,1]$} & 0.005 \\
0.481 & 0.095 & {$[0,1]$} & 0.005 \\
0.515 & 0.095 & {$[0,1]$} & 0.005 \\
\hline
\end{tabular}

TABLE 3: Indoor ammonia measurement belief intervals and probabilities.

\begin{tabular}{lccc}
\hline $\begin{array}{l}\text { Measurement } V_{i} \\
(\mathrm{ppm})\end{array}$ & $\begin{array}{c}\text { Belief interval }=0.95 \\
n=10\end{array}$ & $\begin{array}{c}\text { Possible overall } \\
\text { interval of truth value } V_{\text {it }} \\
(\mathrm{ppm})\end{array}$ & $\begin{array}{c}\text { Basic probability } m\left(V_{\text {it }}\right) \\
\text { of truth value } V_{\text {it }} \\
n=10\end{array}$ \\
\hline 0.604 & 0.095 & {$[0,1]$} & 0.005 \\
0.714 & 0.095 & {$[0,1]$} & 0.005 \\
0.682 & 0.095 & {$[0,1]$} & 0.005 \\
0.687 & 0.095 & {$[0,1]$} & 0.005 \\
0.592 & 0.095 & {$[0,1]$} & 0.005 \\
0.735 & 0.095 & {$[0,1]$} & 0.005 \\
0.523 & 0.095 & {$[0,1]$} & 0.005 \\
0.612 & 0.095 & {$[0,1]$} & 0.005 \\
0.485 & 0.095 & {$[0,1]$} & 0.005 \\
0.491 & 0.095 & {$[0,1]$} & 0.005 \\
\hline
\end{tabular}

TABLE 4: Variance and weighted factors of formaldehyde and ammonia.

\begin{tabular}{lcc}
\hline Gas & Formaldehyde & Ammonia \\
\hline Variances $\sigma_{1 i}^{2}, \sigma_{2 i}^{2}$ & 0.00262 & 0.00505 \\
Weighted factors $\omega_{1 i}, \omega_{2 i}$ & 0.65820 & 0.34180 \\
\hline
\end{tabular}

found that the difference between the level of "serious pollution" and the other levels is quite evident. This conclusion proves that the estimation using RDF algorithm is rather reasonable. Furthermore, the collision degree parameter $C$ is decreased dramatically from 0.01 to 0.0002 .

The evidence collision degree is very important for region-dot conversion fusion (RDF) algorithm. Because if it is extremely severe, $C=1$, the result could not be obtained according to (21). Fortunately, the evidence collision degree was decreased in RDF algorithm compared with D-S approach.

4.4. Comparison. Based on the above process, the experimental results are listed in Table 9. In addition, the performance comparisons are concluded in Table 10. Obviously, the RDF algorithm has improved the separation of the belief interval. Also, the evidence collision degree is decreased dramatically so that the evidential reasoning can continue to be completed.

As for the stability, cost, and complex, the RDF algorithm has a better stability than D-S approach because the evidence collision degree is decreased in RDF algorithm, and it has no extra cost, but it is a little complex than AWF.

\section{Conclusion}

In this paper, the RDF algorithm has been developed to implement the indoor air quality estimation successfully. Firstly, the RD function is defined and the belief interval is converted to a specific value. The $\mathrm{RD}$ function is then combined with the D-S evidential reasoning to complete the RDF processing. The RDF algorithm estimates accurately the indoor air quality because it solves the evidence collision degree problem. Moreover, the separability of the basic belief functions is improved. As can be seen, the original belief function values of formaldehyde were $0.01,0.01,0.29$, and 0.68 for the 4 belief intervals, respectively; and the original 
TABLE 5: Belief intervals of formaldehyde and ammonia.

\begin{tabular}{lcccc}
\hline Item & No pollution & Light pollution & Medium pollution & Serious pollution \\
\hline Formaldehyde & {$[0,0.05]$} & {$[0,0.05]$} & {$[0.28,0.33]$} & {$[0.67,0.72]$} \\
Ammonia & {$[0,0.05]$} & {$[0,0.05]$} & {$[0.19,0.24]$} & {$[0.76,0.81]$} \\
\hline
\end{tabular}

TABLE 6: Dot values of Belief values of formaldehyde and ammonia.

\begin{tabular}{lccccc}
\hline Item & No pollution & Light pollution & Medium pollution & Serious pollution & Collision degree C \\
\hline Formaldehyde & 0.01 & 0.01 & 0.29 & 0.68 & 0.01 \\
Ammonia & 0.01 & 0.01 & 0.20 & 0.77 & 0.01 \\
\hline
\end{tabular}

TABLE 7: Combinations of formaldehyde and ammonia.

\begin{tabular}{|c|c|c|c|c|c|}
\hline \multirow{2}{*}{$\begin{array}{l}\text { Item } \\
\text { Basic belief value } \\
m\left(V_{i}\right) \text { of ammonia }\end{array}$} & \multicolumn{5}{|c|}{ Basic belief value $m\left(U_{i}\right)$ of formaldehyde } \\
\hline & $\begin{array}{c}\text { No pollution } \\
0.01\end{array}$ & $\begin{array}{c}\text { Light } \\
\text { pollution } \\
0.01\end{array}$ & $\begin{array}{c}\text { Medium } \\
\text { pollution } \\
0.29\end{array}$ & $\begin{array}{c}\text { Serious } \\
\text { pollution } \\
0.68\end{array}$ & $\begin{array}{c}\text { Collision degree } C \\
0.01\end{array}$ \\
\hline $\begin{array}{l}\text { No pollution } \\
0.01\end{array}$ & 0.0001 & 0.0001 & 0.0029 & 0.0068 & 0.0001 \\
\hline $\begin{array}{l}\text { Light pollution } \\
0.01\end{array}$ & 0.0001 & 0.0001 & 0.0029 & 0.0068 & 0.0001 \\
\hline $\begin{array}{l}\text { Medium pollution } \\
0.20\end{array}$ & 0.0020 & 0.0020 & 0.0580 & 0.1360 & 0.0050 \\
\hline $\begin{array}{l}\text { Serious pollution } \\
0.77\end{array}$ & 0.0077 & 0.0077 & 0.2233 & 0.5236 & 0.0050 \\
\hline $\begin{array}{l}\text { Collision degree } C \\
0.01\end{array}$ & 0.0001 & 0.0001 & 0.0029 & 0.0068 & 0.0001 \\
\hline
\end{tabular}

TABLE 8: Fusion results of formaldehyde and ammonia.

\begin{tabular}{lccccc}
\hline Item & No pollution & Light pollution & Medium pollution & Serious pollution & Collision degree C \\
\hline formaldehyde & 0.01 & 0.01 & 0.29 & 0.68 & 0.01 \\
ammonia & 0.01 & 0.01 & 0.20 & 0.77 & 0.01 \\
Fusion value & 0.0002 & 0.0002 & 0.0996 & 0.8998 & 0.0002 \\
\hline
\end{tabular}

TABLE 9: Fusion result comparison between AWF method and RDF method.

\begin{tabular}{lcll}
\hline $\begin{array}{l}\text { Formaldehyde } \\
\text { measurements } \\
(\mathrm{ppm})\end{array}$ & $\begin{array}{c}\text { Ammonia } \\
\text { measurements } \\
(\mathrm{ppm})\end{array}$ & $\begin{array}{c}\text { AWF } \\
(\mathrm{ppm})\end{array}$ & $\begin{array}{c}\text { RDF } \\
\text { (probability value) }\end{array}$ \\
\hline 0.501 & 0.604 & & \\
0.498 & 0.714 & Pollution index & The probabilities of the pollution level belong to the "no \\
0.589 & 0.682 & $I=\widehat{\bar{X}}=0.5662$. & $\begin{array}{l}\text { pollution," "light pollution," "medium pollution," and } \\
\text { "serious pollution" which are } 0.0002,0.0002,0.0996,\end{array}$ \\
0.579 & 0.687 & The pollution level & and 0.8998, respectively. The Collision degree is 0.0002. \\
0.657 & 0.592 & belongs to the serious & The pollution level belongs to the serious pollution. \\
0.527 & 0.735 & pollution. & \\
0.472 & 0.523 & & \\
0.481 & 0.612 & & \\
0.515 & 0.485 & & \\
\hline
\end{tabular}

TABLE 10: Performance comparison between AWF, D-S, and RDF.

\begin{tabular}{lccc}
\hline Item & AWF & D-S & RDF \\
\hline Air quality estimation & Serious pollution & Serious pollution & Serious pollution \\
Separability of basic belief function & Not changed & Normal & Better \\
Evidence collision degree & Not changed & Normal & Lower \\
\hline
\end{tabular}


ammonia values were $0.01,0.01,0.20$, and 0.77 , respectively. Using the proposed scheme, the outcome values become $0.0002,0.0002,0.0996$, and 0.8998 , respectively. The result reveals that the air pollution condition could be accurately classified. In addition, the evidence collision degree was decreased from 0.01 to 0.0002 . Compared with the AWF algorithm and D-S method, the proposed RDF algorithm can provide more correct and effective indoor air quality estimation.

Region-dot conversion fusion algorithm is not only suitable for air quality evaluation but also suitable for other aspects, such as object recognition fusion.

\section{Conflict of Interests}

The authors declare that there is no conflict of interests regarding the publication of this paper.

\section{Acknowledgments}

This research is sponsored by Scientific Research Foundation for Returned Scholars, Ministry of Education of China ([2011]508), and Natural Science Foundation of Shaanxi Province (2011JM8005).

\section{References}

[1] K. Schäfer, S. Emeis, C. Jahn et al., "Athens airport air quality study by remote sensing with DOAS, FTIR, and ceilometer," in Remote Sensing of Clouds and the Atmosphere XIII, vol. 7107 of Proceedings of SPIE, pp. 356-390, September 2008.

[2] G. Z. Xiao, Z. Y. Zhang, J. Weber et al., "Trace amount formaldehyde gas detection for indoor air quality monitoring," in Proceedings of the IEEE International Instrumentation and Measurement Technology Conference, pp. 1418-1421, May 2011.

[3] U. S. Akshath, L. Sagaya Selvakumar, and M. S. Thakur, "Detection of formaldehyde in food samples by enhanced chemiluminescence," Analytical Methods, vol. 4, no. 3, pp. 699-704, 2012.

[4] I. K. Panagopoulos, A. N. Karayannis, P. Kassomenos, and K. Aravossis, "A CFD simulation study of VOC and formaldehyde indoor air pollution dispersion in an apartment as part of an indoor pollution management plan," Aerosol and Air Quality Research, vol. 11, no. 6, pp. 758-762, 2011.

[5] O. Basir, F. Karray, and H. W. Zhu, "Connectionist-based Dempster-Shafer evidential reasoning for data fusion," IEEE Transactions on Neural Networks, vol. 16, no. 6, pp. 1513-1530, 2005.

[6] Y. Deng and W. Shi, "Experts' knowledge fusion in modelbased diagnosis based on bayes networks," Journal of Systems Engineering and Electronics, vol. 14, no. 2, pp. 25-30, 2003.

[7] Q. Zhang and G. Wang, "The uncertainty measure of hierarchical quotient space structure," Mathematical Problems in Engineering, vol. 2011, Article ID 513195, 16 pages, 2011.

[8] M. F. Wang, M. E. Zhu, and S. J. Yu, "Multi-sensors data fusion algorithm of improved Dempster-Shafer evidential reasoning," in Proceedings of the International Conference on Signal and Information Processing, pp. 212-214, 2010.
[9] Y. H. Li, B. Vucetic, A. Santoso, and Z. Chen, "Space-time trellis codes with adaptive weighting," Electronics Letters, vol. 39, no. 25, pp. 1833-1834, 2003.

[10] M. Zhang, Z. Liu, H. Zhou, and J. Wang, "From pixels to region: a salient region detection algorithm for location-quantification image," Mathematical Problems in Engineering, vol. 2014, Article ID 826068, 7 pages, 2014.

[11] I. Nevat, G. W. Peters, and I. B. Collings, "Distributed detection in sensor networks over fading channels with multiple antennas at the fusion centre," IEEE Transactions on Signal Processing, vol. 62, no. 3, pp. 671-683, 2014.

[12] A. Assa and F. Janabi-Sharifi, "A robust vision-based sensor fusion approach for real-time pose estimation," IEEE Transactions on Cybernetics, vol. 44, no. 2, pp. 217-227, 2014.

[13] J.-B. Yang and D.-L. Xu, "Nonlinear information aggregation via evidential reasoning in multiattribute decision analysis under uncertainty," IEEE Transactions on Systems, Man, and Cybernetics A: Systems and Humans, vol. 32, no. 3, pp. 376-393, 2002.

[14] M. Menzies and J. Hihn, "Evidence-based cost estimation better-quality for software," IEEE Software, vol. 23, no. 4, pp. 6466, 2006.

[15] S. Démotier, W. Schön, and T. Denœux, "Risk assessment based on weak information using belief functions: a case study in water treatment," IEEE Transactions on Systems, Man and Cybernetics C: Applications and Reviews, vol. 36, no. 3, pp. 382396, 2006.

[16] F. Cuzzolin, "Two new Bayesian approximations of belief functions based on convex geometry," IEEE Transactions on Systems, Man, and Cybernetics B: Cybernetics, vol. 37, no. 4, pp. 993-1008, 2007.

[17] K. G. Kyriakopoulos, F. J. Aparicio-Navarro, and D. J. Parish, "Manual and Automatic assigned thresholds in multi-layer data fusion intrusion detection system for 802.11 attacks," IET Information Security, vol. 8, no. 1, pp. 42-50, 2014.

[18] M. Talha and R. Stolkin, "Particle filter tracking of camouflaged targets by adaptive fusion of thermal and visible spectra camera data," IEEE Sensors Journal, vol. 14, no. 1, pp. 159-166, 2014.

[19] X. Kong and J. Sun, "Adaptive weight particle filter for nor-linear noisy signals," in Proceedings of the 7th International Conference on Natural Computation (ICNC '11), vol. 2, pp. 677-680, July 2011.

[20] H. Zhang, H. Wu, and L. Lu, "Analysis and algorithm for robust adaptive cooperative spectrum-sensing," IEEE Transactions on Wireless Communications, vol. 13, no. 2, pp. 618-629, 2014.

[21] Z. Q. Cai and H. Huang, "Ant colony optimization algorithm based on adaptive weight and volatility parameters," in Proceedings of the 2nd International Symposium on Intelligent Information Technology Application (IITA '08), vol. 3, pp. 75-79, December 2008.

[22] E. Bort, M. Donelli, A. Martini, and A. Massa, "An adaptive weighting strategy for microwave imaging problems," IEEE Transactions on Antennas and Propagation, vol. 53, no. 5, pp. 1858-1862, 2005.

[23] M. K. Kang, D. Y. Kim, and K. J. Yoon, "Adaptive support of spatial-temporal neighbors for depth map sequence upsampling," IEEE Signal Processing Letters, vol. 21, no. 2, pp. 150154, 2014.

[24] Y. Leung, J. Liu, and J. Zhang, "An improved adaptive intensityhue-saturation method for the fusion of remote sensing images," IEEE Geoscience and Remote Sensing Letters, vol. 11, no. 5, pp. 985-989, 2014. 
[25] W. H. Lee, K. Choi, and J. B. Ra, "Frame rate up conversion based on variational image fusion," IEEE Transactions on Image Processing, vol. 23, no. 1, pp. 399-412, 2014.

[26] A. S. Gebregiorgis and F. Hossain, "Estimation of satellite rainfall error variance using readily available geophysical features," IEEE Transactions on Geoscience and Remote Sensing, vol. 52, no. 1, part 1, pp. 288-304, 2014.

[27] B. Liu, Y. Li, T. Wang, F. Shen, and Z. Bao, "An analytical formula approximating the multilook interferometric-phase variance for InSAR," IEEE Geoscience and Remote Sensing Letters, vol. 11, no. 4, pp. 878-882, 2014.

[28] E. Eweda, "Dependence of the stability of the least mean fourth algorithm on target weights non-stationarity," IEEE Transactions on Signal Processing, vol. 62, no. 7, pp. 1634-1643, 2014. 


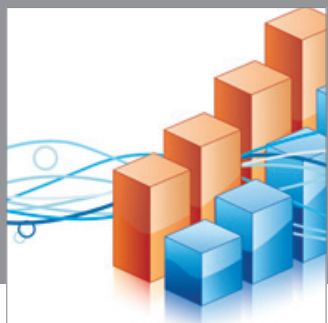

Advances in

Operations Research

mansans

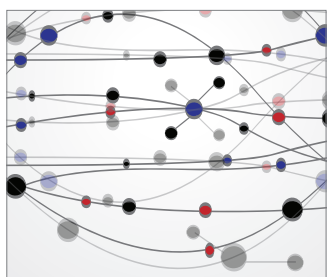

The Scientific World Journal
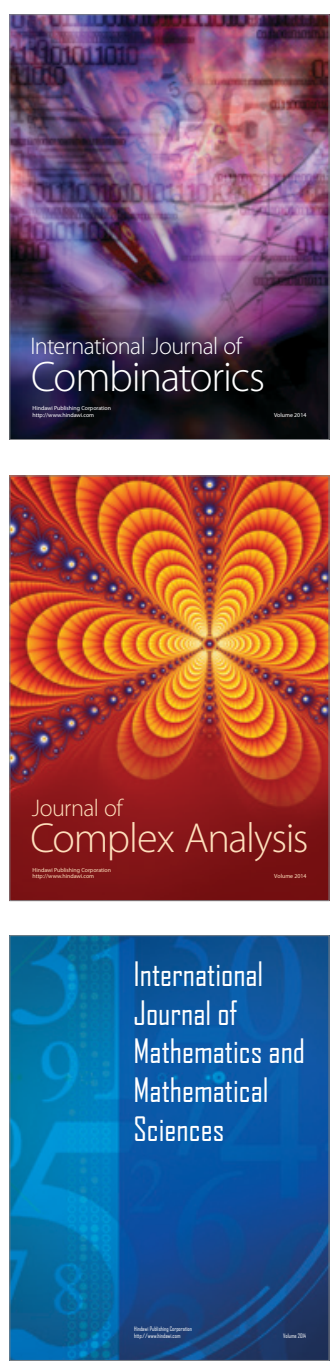
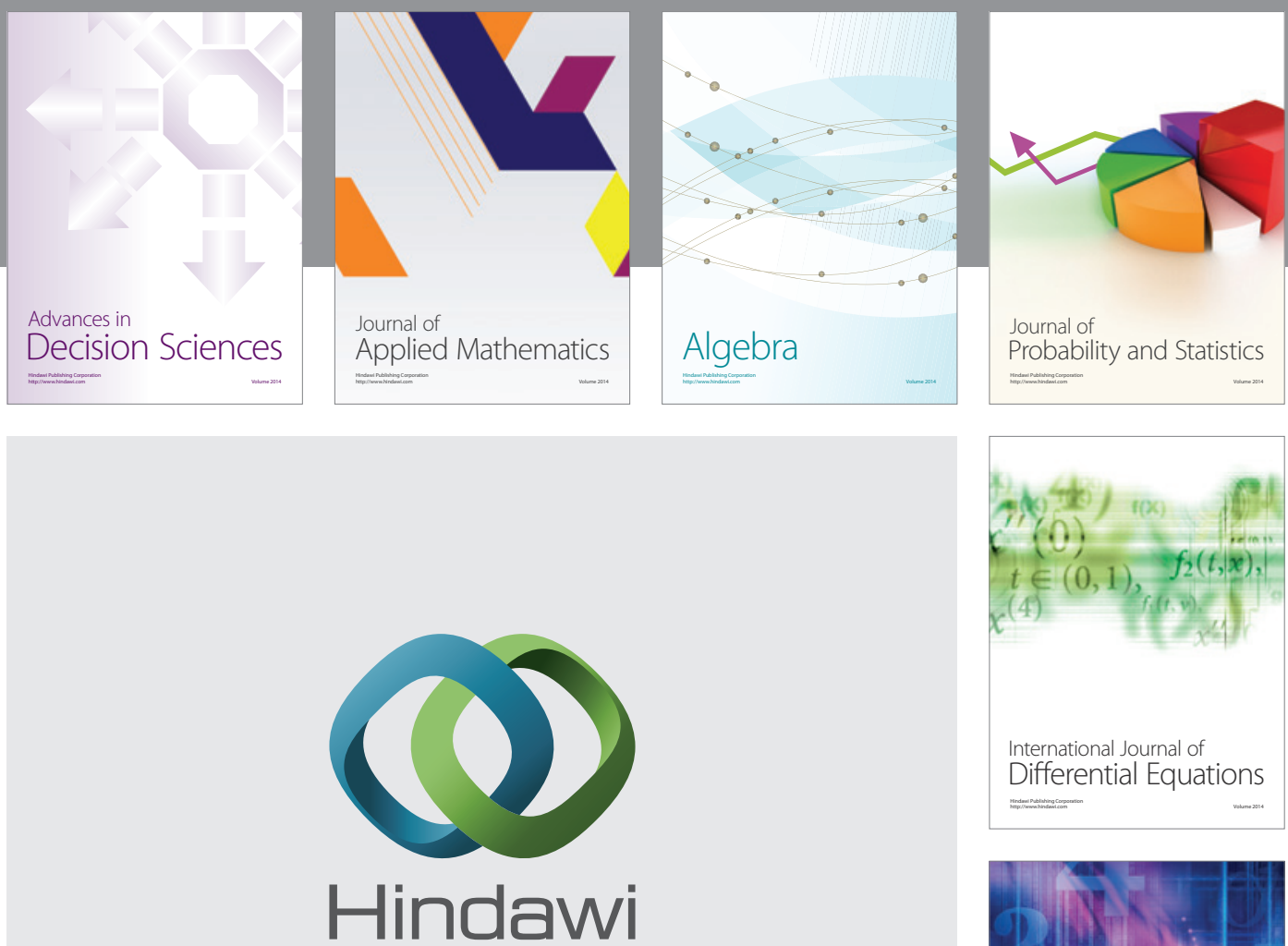

Submit your manuscripts at http://www.hindawi.com
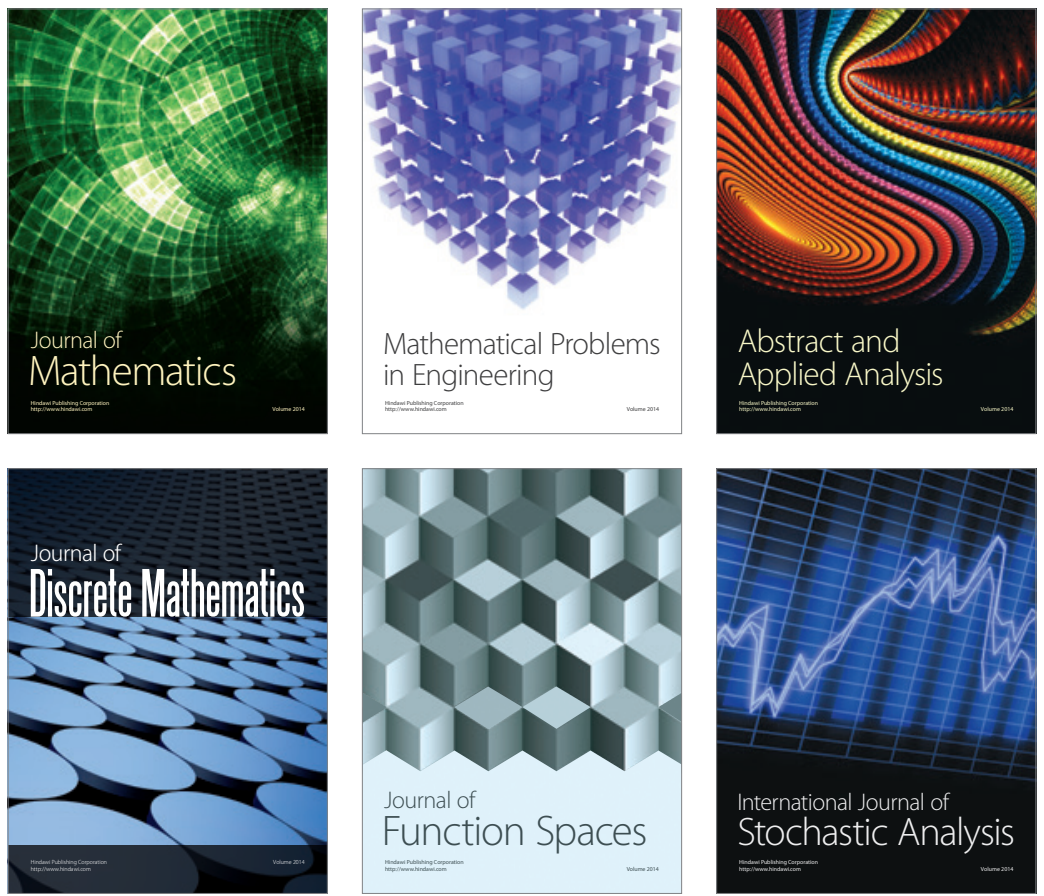

Journal of

Function Spaces

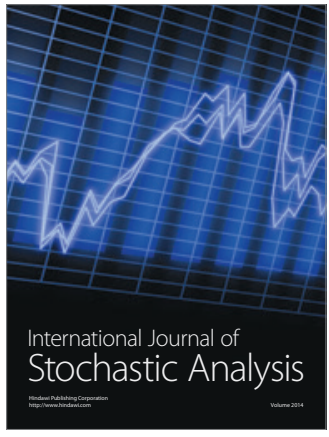

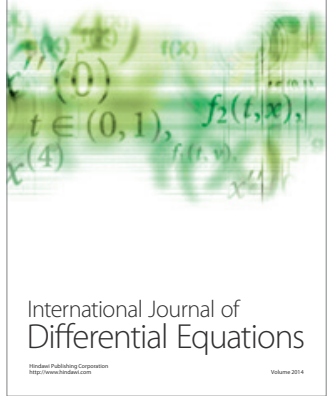
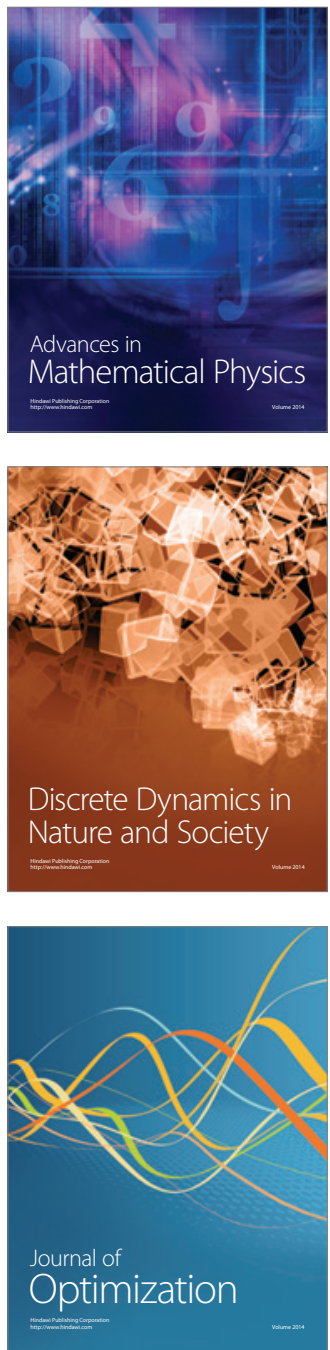